\title{
New Approach for Direct Chemical Synthesis of Hexagonal Co Nanoparticles
}

\author{
Frank M. Abel ${ }^{1 *}$, Vasilis Tzitzios ${ }^{2}$ and George C. Hadjipanayis ${ }^{1}$ \\ ${ }^{1}$ Physics and Astronomy, University of Delaware \\ ${ }^{2}$ Institute of Nanoscience and Nanotechnology, NCSR, Demokritos, Greece \\ fabel@udel.edu
}

\begin{abstract}
In this paper, we explore the possibility of producing hexagonal Cobalt nanoparticles, with high saturation magnetization by direct chemical synthesis. The nanoparticles were synthesized by reduction of anhydrous cobalt (II) chloride by $\mathrm{NaBH}_{4}$ in tetraglyme at temperatures in the range of 200 to $270^{\circ} \mathrm{C}$ under a nitrogen-hydrogen atmosphere. The reactions were done at high temperatures to allow for the formation of as-made hexagonal cobalt. The size of the particles was controlled by the addition of different surfactants. The best magnetic properties so far were obtained on spherical hexagonal Co nanoparticles with an average size of $45 \mathrm{~nm}$, a saturation magnetization of $143 \mathrm{emu} / \mathrm{g}$ and coercivity of 500 Oe. The saturation magnetization and coercivity were further improved by annealing the Co nanoparticles leading to saturation magnetization of $160 \mathrm{emu} / \mathrm{g}$ and coercivity of 540 Oe.
\end{abstract}

\section{Introduction}

Magnetic nanoparticles have attracted much interest in recent years due to their interesting and unique magnetic properties, when compared to bulk materials. These properties make them attractive candidates for a large range of technological applications, such as biomedical [1,2], recording media [3], magnetic fluids [4], and as catalysts [5]. As is well known, the magnetic properties are strongly dependent on particle size and processing and therefore a complete scientific understanding of their properties, and the techniques of synthesis is necessary to fully exploit their potential. Cobalt is a well-

(C) 2015. This manuscript version is made available under the Elsevier user license

http://www.elsevier.com/open-access/userlicense/1.0/ 
known ferromagnetic material with high saturation magnetization and can exist in both the face centered cubic phase (fcc), and the hexagonal structure (hcp). The cubic phase exhibits soft properties while the hexagonal phase can exhibit some coercivity due to the moderate value of magnetocrystalline anisotropy of $\mathrm{K}_{\mathrm{a}}=4.5 \times 10^{5} \mathrm{~J} / \mathrm{m}^{3}[6]$ of this phase.

Previous wet chemical methods such as thermal decomposition of metal precursors, sonochemical, solvothermal process, metal salt reduction, reverse micelles and polyol process [7-14] have been shown to produce low dimensional single crystal cobalt. In this study, we have investigated a new wet chemical method for producing crystalline Cobalt nanoparticles. This method involves high temperature reduction of metal salts in tetraglyme by $\mathrm{NaBH}_{4}$, and is of interest because it can produce directly crystalline hcp Cobalt with high saturation magnetization (143 emu/g), close to that of bulk and with a moderate coercivity of 500 Oe while on the nanoscale (20 to $100 \mathrm{~nm}$ ).

\section{Experiment}

The synthetic procedure is based on the reduction of anhydrous cobalt (II) chloride by sodium borohydride at elevated temperatures $\left(200-270^{\circ} \mathrm{C}\right)$ in tetraglyme under a nitrogen hydrogen atmosphere. In more details. The synthesis was carried out by dissolving the $\mathrm{CoCl}_{2}$ in $15 \mathrm{~mL}$ of tetraglyme in addition to one of the following surfactants oleylamine, oleic acid, and polyvinylpyrrolidone, in a $100 \mathrm{~mL} 3$ stopper flask under a $5 \% \mathrm{H}_{2}-95 \% \mathrm{Ar}$ atmosphere, and purging continuously which creates a light blue solution. A second solution of $5 \mathrm{~mL}$ tetraglyme and varying amounts of $\mathrm{NaBH}_{4}$ was formed, using ultra sonication to dissolve the $\mathrm{NaBH}_{4}$ and then purging with $5 \% \mathrm{H}_{2}-95 \%$ Ar, creating a clear white solution. The solution of $5 \mathrm{~mL}$ tetraglyme and $\mathrm{NaBH}_{4}$ was then injected directly into the solution contained in the $100 \mathrm{~mL} 3$-stopper flask at the refluxing temperature, causing the solution to turn from blue to black; the best results were found by injecting the $\mathrm{NaBH}_{4}$ at $270^{\circ} \mathrm{C}$. After the addition of the $\mathrm{NaBH}_{4}$ the solution was held at the refluxing temperature for 5 minutes. The solution was then allowed to cool to room temperature. The black precipitation was magnetically separated from the solution and washed with, ethanol, acetone, hexane, and distilled water depending on the stabilizing agent. The particles were 
washed repeatedly with one of the above solvents, using high ultra-sonication and magnetic separation until the surfactants were removed. The black powder was then dried at room temperature in air.

The as-made particles were further annealed under a $5 \% \mathrm{H}_{2}-95 \% \mathrm{Ar}$ atmosphere to improve their properties. The best results were obtained for a short annealing of 30 minutes at $440^{\circ} \mathrm{C}$, with minimal increase in particle size. The materials was fully was characterized by X-raydiffraction (XRD), scanning electron microscopy (SEM), and a vibrating sample magnetometry (VSM).

\section{Results and Discussion}

Figure 1 shows the X-ray diffraction patterns of the as-made materials synthesized with different surfactants (oleylamine, oleic acid, and polyvinylpyrrolidone) and the annealed oleic acid sample at $440^{\circ} \mathrm{C}$ for 30 minutes. The X-ray diffractions patterns show the three primary peaks at $41^{0}, 44.7^{0}$ and $47^{0}$ which are indexed with the hcp cobalt phase corresponding to the (101), (002), (100) diffractions. The dominant phase in all four diffraction patterns appears to be hcp Cobalt, which as discussed later is in agreement with the magnetic properties as shown in Figure 2. The presence of a small amount of fcc phase can't be excluded especially in the oleic acid sample Fig. 1 a) as seen by the very small (111) and (002) peaks of fcc phase. 

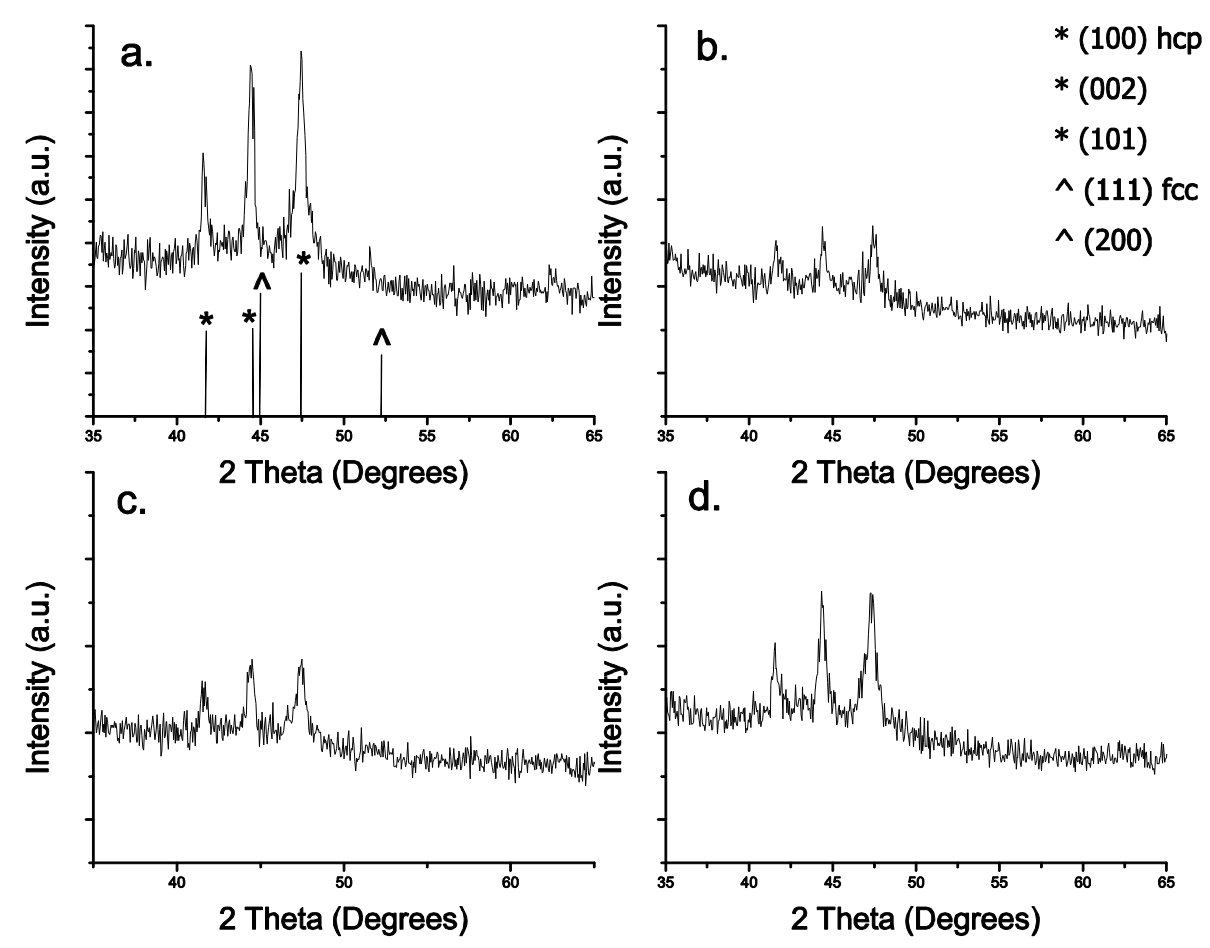

Figure 1. X-ray diffraction patterns from Co nanoparticles synthesized in different surfactants and after annealing a) oleic acid b) annealed oleic acid Sample c) oleylamine d) polyvinylpyrrolidone

The room temperature hysteresis loops of the corresponding samples are shown in Fig. 2. The saturation magnetization values of as-made samples are in the range of $111 \mathrm{emu} / \mathrm{g}$ to $143 \mathrm{emu} / \mathrm{g}$ with nearly the same values of coercivity around 500 Oe with the largest values obtained in the oleic acid sample as shown in Fig. 2 a). Although, the other surfactants produced the same crystal phase the oleic acid gave the best crystallinity as seen in Fig. 1. The sample prepared in oleic acid was then annealed under $5 \% \mathrm{H}_{2}-95 \%$ Ar at $440^{\circ} \mathrm{C}$ for 30 minutes giving a magnetization of $(160 \mathrm{emu} / \mathrm{g})$ which is similar to bulk Co, and a slightly improved coersivity (540 Oe) as shown in Fig. 2 b). The different magnetic properties of the samples, even though they exhibit the same crystalline phase as can be seen in Figure 1 , can be explained mainly by the different particle sizes and the remnant surfactant on the particles. 
Each of the different surfactants effects particle growth and subsequently the size the resulting particles which can have a direct effect on the coercivity and magnetization when dealing with nanoscale materials as seen in Figure 3. Additionally, the remaining amount of surfactants on the nanoparticles can greatly affect their saturation magnetization. Oleic acid can be easily removed from the resultant material, while polyvinylpyrrolidone can be extremely difficult to remove leading to organic material in the end product which explains the much lower saturation magnetization of this sample.
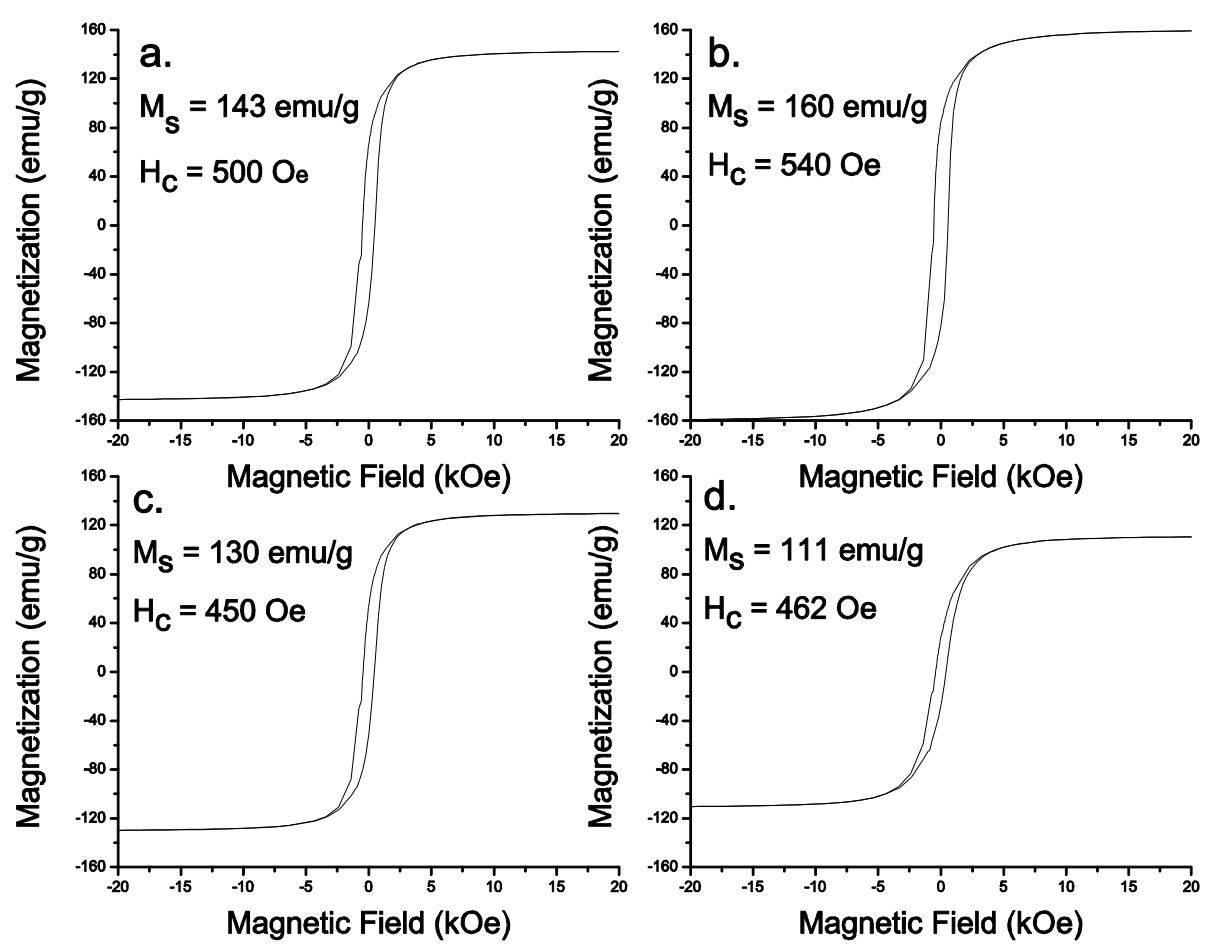

Figure 2. Room temperature hysteresis loops from Co particles synthesized in different surfactants and after annealing a) oleic acid b) annealed oleic acid sample c) oleylamine d) polyvinylpyrrolidone

Figure 3 shows the particle morphology as seen by SEM. The oleic acid and oleylamine surfactants used in their respective quantities produced spherical Co nanoparticles with similar size and particle distribution as shown in Fig. 3 a) and c) ranging from 20 to $100 \mathrm{~nm}$. The sample prepared in polyvinylpyrrolidone Fig. 3 d) have an even greater particle distribution ranging from 10 to $150 \mathrm{~nm}$ in size, and exhibit more square shaped particles compared to the samples showin in Fig. 3 a) and c). 
Figure 3 also shows the microstuctructure of the oleic acid sample after annealing. The overall shape remained the same, but the particle size increased with some sintering occuring as expected. However, the particles still remain less than $150 \mathrm{~nm}$ in size.
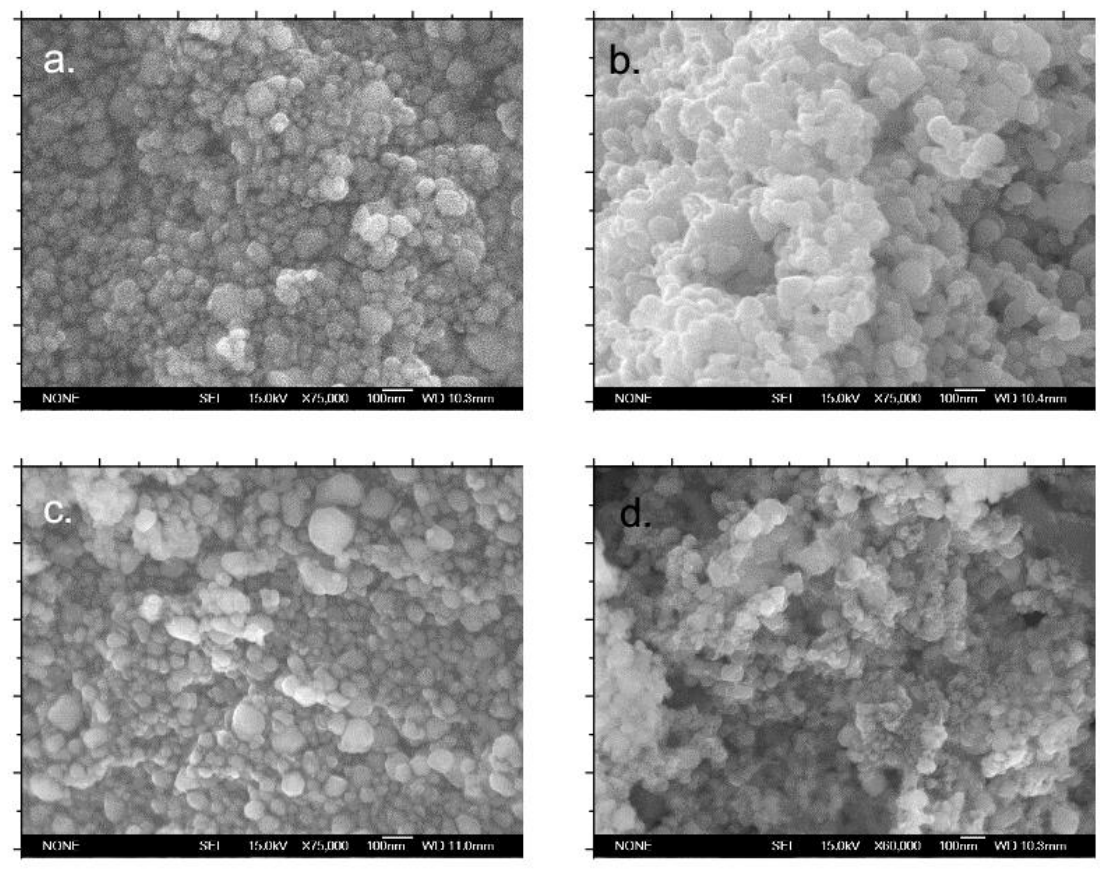

Figure 3. SEM of as-synthesized Co particles in different surfactants and after annealing a) oleic acid b) annealed aleic acid sample c) aleylamine d) polyvinylpyrrolidone

\section{Conclusion}

A facile chemical approach to the suctrural selective synthesis of spherical hcp Co nanoparticles is presented. The hexagonal phase can be obtained directly by high temperature chemical synthesis in the temperatire range of 200 to $270^{\circ} \mathrm{C}$ using $\mathrm{NaBH}_{4}$ as a reducing agent in tetraglyme. Although higher coercivty values have been reported $[8,15]$, none have been able to obtain moderate coercivity and 
saturation magnetization values close to that of bulk Co. We have shown that spherical Co particles can be synthesized with a high magnetization $(143 \mathrm{emu} / \mathrm{g})$ and with a moderate coercivity of around (500 Oe). After annealing a saturation magnetization of (160 emu/g) and coercivity of (540 Oe) is obtained.

\section{Acknowledgements}

Work supported by DOE-BES-DMSE, DE-FG02-04ER4612.

\section{References}

[1] Q.A. Pankhurst, J. Connolly, S.K. Jones, and J. Dobson, Applications of magnetic nanoparticles in biomedicine, J.

Phys. D: Appl. Phys. 36 2003, pp. R167-R181.

[2] K.B. Lee, S. Park, and C.A. Mirkin, Multicomponent magnetic nanorods for biomolecular separations, Angew. Chem. Int. Edn. 43 2004, pp. 3048- 3050.

[3] S. Sun, C.B. Murray, D. Weller, L. Folks, and A. Moser, Monodisperse FePt nanoparticles and ferromagnetic FePt nanocrystal superlattices, Science 287 (2000), pp. 1989-1992.

[4] ] M.P. Pileni, The role of soft colloidal templates in controlling the size and shape of inorganic nanocrystals, Adv. Funct. Mater. 11 (2001), pp. 323-333.

[5] J. Park, E. Kang, S.U. Son, H.M. Park, M.K. Lee, J. Kim, K.W. Kim, H.J. Noh, J.H. Park, C.J. Bae, J.G. Park, and T. Hyeon, Monodisperse nanoparticles of Ni and NiO: synthesis,

[6] Ram S. Mater. Sci. A304-306 2001 923-927

[7] Diana F. S., Lee S. H., Petroff P. M., and Kramer E. J. Nano Letters 2003 Vol. 3, No. 7, 891- 895

[8] Soumare Y., et. Al. Adv. Funct. Mater. 2009, 19, 1971-1977

[9] Puntes V. F., Zanchet D., Erdonmez C. K., and Alivisatos P. A. J. AM. Chem. Soc. 2002, 124, 12874- 12880

[10] Zhang Z., Chen X., Zhang X., and Shi C. Solid State Comm. 1392006 403- 405

[11] de la Pena O' Shea V. A., et. Al. Chem. Mater. 2009, 21, 5637- 5643

[12] Chakroune N., Viau G., Ricolleau C., Fievet-Vincent F., and Fievet F. J. Mater. Chem, 2003, 13, 312-318

[13] Srinivasa Rao K., et. al. J. of Experimental Nanoscience, 2013 Vol. 8. No. 2, 162- 170

[14]Xie By-Qin, et. al. Eur. J. Inorg. Chem. 2006, 2454- 2459

[15] Grandha K., Elkins K. Poudyal N., Liu X., and Ping Liu X. Scientific Reports 4, 53452014 